\title{
Erratum to: Intellectual disability health content within medical curriculum: an audit of what our future doctors are taught
}

\author{
Julian N. Trollor ${ }^{1 *}$, Beth Ruffell ${ }^{1}$, Jane Tracy ${ }^{2,3}$, Jennifer J. Torr ${ }^{2,3}$, Seeta Durvasula ${ }^{4}$, Teresa lacono ${ }^{5}$,
} Claire Eagleson ${ }^{1}$ and Nicolas Lennox ${ }^{6}$

\section{Erratum}

After publication of the original article [1], the authors found two errors within the Results section of the Abstract. Details are as follows:

1) In the line 'Elective content varies markedly across universities ( 1 to $122 \mathrm{~h}$ ), the number " 122 " should be " 222 ".

2) In the line 'In Australia, medical school curricula contain a median of $2.55 \mathrm{~h}$ of compulsory intellectual disability content', there should be a word "units" inserted between "curricula" and "contain". The full corrected sentence should read: 'In Australia, medical school curricula units contain a median of $2.55 \mathrm{~h}$ of compulsory intellectual disability content'.

\begin{abstract}
Author details
'Department of Developmental Disability Neuropsychiatry (3DN), UNSW Australia, 34 Botany Street, Randwick 2052, NSW, Australia. ${ }^{2}$ Centre for Developmental Disability Health Victoria (CDDHV), Monash Health, 122 Thomas Street, Dandenong 3175, VIC, Australia. ${ }^{3}$ Faculty of Medicine, Nursing and Health Sciences, Monash University, Melbourne 3800, VIC, Australia. ${ }^{4}$ Centre for Disability Studies, Sydney Medical School, The University of Sydney, Level 1, Medical Foundation Building, 92-94 Parramatta Road, Camperdown 2050, NSW, Australia. ${ }^{5}$ La Trobe Rural Health School, La Trobe University, PO Box 199, Bendigo 3552, VIC, Australia. ${ }^{6}$ Queensland Centre for Intellectual and Developmental Disability (QCIDD), Mater Research Institute, The University of Queensland, Level 2 Aubigny Place, Mater Hospitals, South Brisbane QLD 4101, Australia.
\end{abstract}

Published online: 05 October 2016

\section{Reference}

1. Trollor JN, et al. Intellectual disability health content within medical curriculum: an audit of what our future doctors are taught. BMC Med Educ. 2016;16:105. doi:10.1186/s12909-016-0625-1.

\footnotetext{
* Correspondence: j.trollor@unsw.edu.au

'Department of Developmental Disability Neuropsychiatry (3DN), UNSW

Australia, 34 Botany Street, Randwick 2052, NSW, Australia
} 